\title{
Impact of Average-Dwell-Time Characterizations for Switched Nonlinear Systems on Complex Systems Control
}

\author{
Honoring Academician Vsevolod M. Kuntsevich for his life-long \\ contributions to Control Systems Theory and Applications
}

\author{
Georgi Dimirovski ${ }^{1,2}$ \\ ${ }^{1}$ Faculty of Engineering, Dogus University of Istanbul \\ Acibadem, Istanbul 34722, Rep. of Turkey \\ ${ }^{2}$ FEIT - Karpos 2, St. Cyril and St. Methodius University \\ Skopje 1000, Rep. of Macedonia; Email: gdimirovski@dogus.edu.tr \\ Jiqiang Wang ${ }^{3}$ \\ ${ }^{3}$ Jiangsu Province Key Laboratory of Aerospace Power Systems \\ Nanjing University of Aeronautics \& Astronautics \\ Nanjing 210016, P. R. of China; Email: jiqiang.wang@nuaa.edu.cn \\ Hong Yue ${ }^{4}$ \\ ${ }^{4}$ Department of Electronic \& Electrical Engineering \\ University of Strathclyde, Glasgow, Scotland, UK \\ Email: hong.yue@strath.ac.uk \\ Jovan Stefanovski ${ }^{5}$ \\ ${ }^{5}$ J.P. "Strezhevo", Division of Control \& Informatics, \\ Ul. 1 Maj BB, Bitola 6000, R. Macedonia; Email: jovanstef@t.mk
}

\begin{abstract}
It is well known, present day theory of switched systems is largely based on assuming certain small but finite time interval termed average dwell time. Thus it appears dominantly characterized by some slow switching condition with the average dwell time satisfying a certain lower bound, which implies a constraint nonetheless. In cases of nonlinear systems, there may well appear certain non-expected complexity phenomena of particularly different nature when switching becomes no longer useful. A fast switching condition with average the dwell time satisfying an upper bound is explored and established. A comparison analysis of these innovated characterizations via slightly different overview yielded new results on the transient behaviour of switched nonlinear systems, while preserving the system stability. The approach of multiple Lyapunov functions is used in current analysis and the switched systems framework is believed to be extended slightly. Thus some new insight into the underlying, switching caused, system's complexities has been achieved.
\end{abstract}




\section{Introduction}

Inspired by the work [31] of Ye and co-authors (1998), recently, in 2017 the authors of work [8] have extended considerably their previous findings [31] so as to open new insights and light on the underlying system complexities induced by average dwell-time switching law in conjunction with traditional feedback control strategies. Now these authors have privilege to present their most recent extension in order to honour Academician Vsevolod M. Kuntsevich (in the middle on Fig. 1) for his many contributions to Control Systems Theory and Applications [13-16]. In particular, the first author especially honors his past learning the refined usage of Lyapunov functions stability analysis and control synthesis from the 1977 monograph "Sintez sistem avtomaticheskogo upravleniya s pomoshtyu funkciy Lyaponova” by V. M. Kunstevich and M. M. Lychak [14].

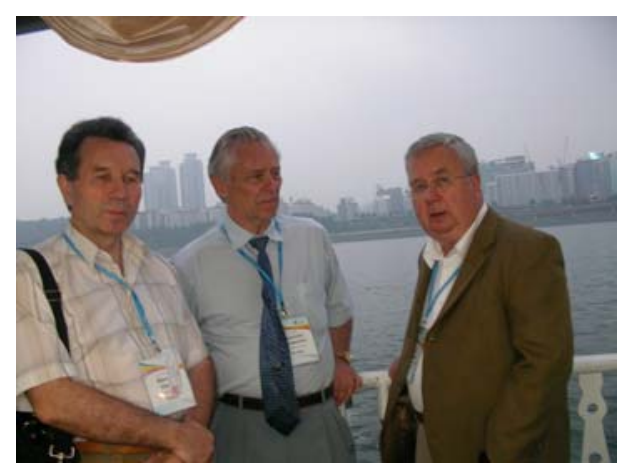

Figure 1 V. Kuntsevich at the IFAC World Congress in Seoul, R. Korea in 2008, in the middle along with his colleagues Academicians S. Vassilev, to the left, and A. Kurzanskiy, to the right (courtesy by our good common friend the late S. Zemlyakov, may he rests in peace).

The authors' innovated extension of the Average-Dwell-Time (ADT) switched systems and control theory is intuitively considerably interesting and appealing too, since it could often yield reducing the conservatism of stability results in the applications. This may not be amenable even to techniques that employ the multiple Lyapunov functions approach [3]. It was shown in [8] both the slow switching and the fast switching can be studied within an appropriately redefined framework. These implications were further worked out in producing novel results on stability of switched nonlinear systems, which are further refined in here. In addition, appropriate simulations are given in this paper to illustrate those results.

It is well known [13] that the behaviour of general nonlinear dynamic systems [7], Fig. 2, and of hybrid systems in particular, to which also switched systems also belong to, may have remarkably different system dynamics from either of their components $[3,5,6,11,12,19,20,21]$. In the case of switched systems for instance, one switched system can be stable although all its components are unstable, also, some inappropriate switching signal may destabilize the overall switched system even though all of its components are stable [3, 22, 26, 28-32]. The initial opening research [27] on switched systems and control was due to D. D. Sworder 
(1976), who investigated the control of systems subject to sudden changes. This appeared as an alternative to the theory of variable structure control systems [9] due to S. V. Emalyanov (1967). In more recent literature, following the results by A. P. Molchanov and Y. S. Pyatnitsky [25] in 1989 and by A. S. Morse [26] in 1996, 1997, a switched system is defined as a rather important category of hybrid systems that consists of a family of continuous-time or discrete-time subsystems and a switching law which governs the switching between the subsystems. During the last decade, considerable attention has been paid to switched systems; in due times, many important results have been obtained since the pioneering contributions of A. S. Morse $(1996,1997)$ and M. S. Branicky (1998); for instance, see [2, $4,15,16,21,22,28-30]$ and [36-43] and references therein. Given the fundamental crucial property of stability in systems engineering, all approaches based on analytical studies of switched systems and switching based control have relied on Lyapunov stability theory and its various extensions; see all given references and references therein. Naturally, Lyapunov stability theory [1, 2, 13-16, 25] and its extensions were instrumental for building up the theory of switched systems and switching based control $[8,11,12,21,23,26]$. However, the issue of system stability in the case of switched systems appeared to be rather delicate one $[8,11,22$, 26, 36-43].

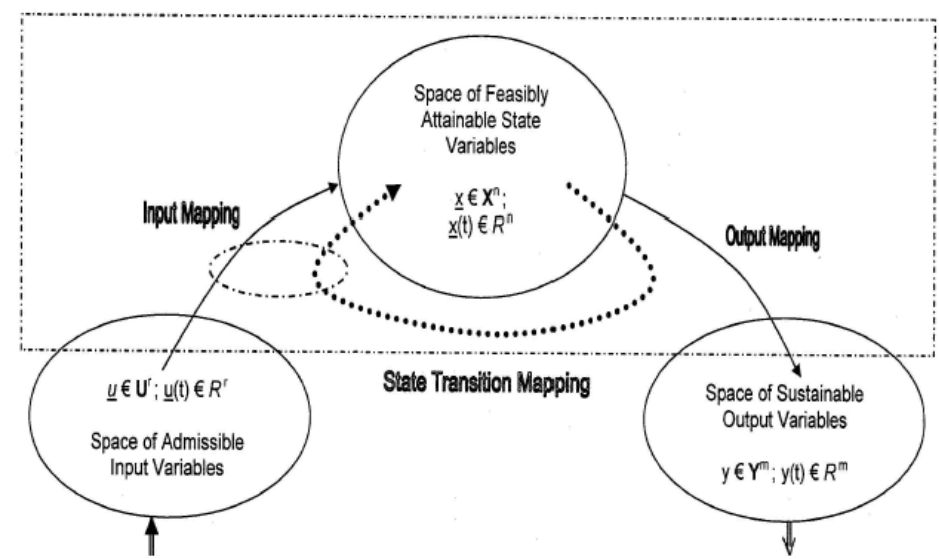

Figurer 2 Illustration of controlled, general nonlinear, systems in accordance to the fundamental laws of physics [7, 8]. Although input, state and output spaces in terms of involved classes of functions can be mathematically defined by a chosen measuring norm, all the vector-valued variables become real-valued vectors at any fixed instant of time, and norm employed is Euclidean one most often.

Most of the existing literature about switched systems is focused on the problem of stability under arbitrary switching [22, 25, 36]. In order to guarantee stability under arbitrary switching, the common Lyapunov function method plays a rather important role (if not the central role because of its conservatism). This is because the existence of a common Lyapunov function implies the global uniform asymptotic stability of the switched system. The importance of common Lyapun- 
ov function has been further consolidated by a converse theorem [22] due to Molchanov and Pyatnitskiy (1989), that asserted if the switched system is globally uniformly asymptotically stable (GUAS), then all the subsystems ought to have a common Lyapunov function.

Over time and in particular more recently, the approach exploiting multiple Lyapunov functions [3] and the associated dwell time [11, 17, 18] or the average dwell time alone [20] are recognized as rather efficient tools in stability studies of switched nonlinear systems [17, 32, 39-43]. The concept of average dwell time switching, which was introduced by Hespanha and Morse (1999) in [12], appeared more general than the standard dwell time switching for both stability analysis and related control design and synthesis problems; for instance, see [32-43]. It does imply that the number of switching actions in a finite interval is bounded from above while the average time between two consecutive switching actions is not less than a constant. It is believed the multiple Lyapunov function approach per-se reduces the inherent conservatism of the common Lyapunov function approach.

In fact, when confining to dynamic systems only, some well-known design procedures for explicit construction of multiple Lyapunov functions have been developed among which the S-procedure and the methodology of Linear Matrix Inqualities (LMI) have been particularly fruitful as most of references in this paper on control synthesis design and the references therein have clearly demonstrated. In multiple Lyapunov functions approaches, it is generally assumed that each Lyapunov-like function associated for each subsystem is increasing (with the first time-derivative decreasing) with time as time elapses. For the first time, Ye and co-authors (1998) in their stability theory for hybrid systems [36] have also studied an approach allowing for a Lyapunov-like function to rise to a limited extent and thus established rather interesting property of a class of such functions called weak Lyapunov.

In order to exploit the concept and results for average-dwell time switched nonlinear systems into control synthesis design, it appeared that it is of paramount importance to keep trace with the application feasibility of Linear Matrix Inequality (LMI) methodology and the S-procedure [33-35] due to of Vladimir A. Yakubovich $(1962,1964,1971)$. In case of other classes of switched systems for that matter the same is equally valid and important, of course. The major reason now is the availability of the respective software tools for LMI [10] and Simulink computing support by Matlab [23, 24]. However, given the well observed fact that typically control synthesis design in practical applications is usually consisted of at least a few iterative cycles of adequate synthesis-simulation-rectifyingmodification $[13,14]$, the importance of consistency with the LMI methodology and S-procedure becomes rather crucial.

A note on notation: The notation used in this work is a fairly standard one. This may well be inferred from: $\mathbb{R}^{n}$ represents the n-dimensional Euclidean space; $C^{2}$ denotes the space of twice continuously differentiable functions; $C^{1}$ once differentiable piece-wise function; $\sigma:\left[t_{0},+\infty\right)$ denotes a causal real-world signal; $M$ 
index set of integers; $\left\{\left(\left[t_{0}, t_{1}\right], \sigma\left(\left[t_{0}, t_{1}\right]\right)\right), \cdots\left(\left[t_{k}, t_{k+1}\right], \sigma\left(\left[t_{k}, t_{k+1}\right)\right), \cdots\right\}\right.$ switching sequence.

\section{Background and Mathematical Preliminaries}

\section{A Couple of Preliminary Notes}

It is important to notice the advanced Lyapunov stability theory, the full account of which is found in Khalil's monograph [13] employing both class $K$ and class $\ll \complement$ functions (additional to the classic Lyapunov functions) as well as his Comparison Principle, is used in this study. The concepts of these functions are restated first for reasons of readability.

Definition 1 [13] A continuous function $k:[0, a) \rightarrow[0, \infty)$ is aid to belong to class $K$ if it is strictly increasing and has value $k(0)=0$. It is said to belong to

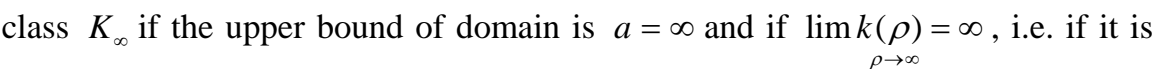
$k:[0, \infty] \rightarrow[0, \infty]$.

Definition 2 [13] A continuous mapping $k=k(\rho, \sigma)$ defined by function $k:[0, a) \times[0, \infty) \rightarrow[0, \infty)$ is said to belong to class $z \mathcal{L}$ if, for each fixed $\sigma$, the mapping $k=k(\rho, \sigma)$ belong to class $K$ functions with respect to $\rho$ and, for each fixed $\rho$, the mapping $k=k(\rho, \sigma)$ is decreasing with respect to $\sigma$ such that $\lim _{\sigma \rightarrow \infty}(\rho, \sigma)=0$.

\section{On Basics of Switched Systems Theory}

It is well known that, in general terms [5, 7, 14, 15], a controlled nonlinear dynamic system can be represented by means of the state transition and output measurement equations (1). In order such a system to have sustained operability functions its state transition mechanism must have the property $f(0,0)=0$ and output measuring mechanism must have $h(0)=0$ on the grounds of basic natural laws.

$$
\left\{\begin{array}{c}
\dot{x}(t)=f(x(t), u(t)), x\left(t_{0}\right)=x_{0} \\
y(t)=h(x(t)) .
\end{array}\right\} \text { for } \forall t \in\left[t_{0},+\infty\right) .
$$

In here, the system's quantities denote: the state space $x \in X^{n}$, the input space $u \in U^{r}, y \in Y^{m}$ the output space; $f: \mathbb{R}^{n} \times \mathbb{R}^{r} \rightarrow \mathbb{R}^{n}, h: \mathbb{R}^{n} \rightarrow \mathbb{R}^{m}$. Upon the synthesis design of a certain controlling infrastructure is carried out then it follows 


$$
u=u\left(t ; t_{0}, u_{0}\right), \forall t \in\left[t_{0}, \infty\right)
$$

Notice that Figure 1 depicts a relevant illustration, which is related to general class of systems and the quoted notions of system theory. For, it depicts the controlled general nonlinear systems in accordance to the fundamental laws of physics on rigid-body energy, matter and momentum of motion, i.e. evolution of natural processes over time.

Furthermore, a controlled nonlinear dynamic system

$$
\left\{\begin{array}{c}
\dot{x}(t)=f_{\sigma}(x(t), u(t)), x\left(t_{0}\right)=x_{0} \\
y(t)=h_{\sigma}(x(t)) .
\end{array}\right\} \text { for } \forall t \in\left[t_{0},+\infty\right),
$$

where subsystems $f_{\sigma(t)}=f_{i}, h_{\sigma(t)}=h_{i}$ with $i \in M$ in an index set $M$ are fixed given models, and the control input $u=u(t)$ is also given (upon its synthesis) is called a switched system. An autonomous switched nonlinear dynamic system thus appears to be defined as follows:

$$
\left\{\begin{array}{c}
\dot{x}(t)=f_{\sigma}(x(t)), x\left(t_{0}\right)=x_{0} \\
y(t)=h_{\sigma}(x(t)) .
\end{array}\right\} \text { for } \forall t \in\left[t_{0},+\infty\right) .
$$

For a causal signal $\sigma:\left[t_{0},+\infty\right)$, if it is

$$
\sigma\left(t^{+}\right)=\Sigma\left\{\left[t_{0}, t\right],\left(\sigma\left[t_{0}, t\right]\right),\left\{\left(x\left(\left[t_{0}, t\right]\right), y\left(\left[t_{0}, t\right]\right)\right\}, \text { for } \forall t \in\left[t_{0},+\infty\right)\right.\right.
$$

a piece-wise constant time-sequence function such that $\sigma\left(t^{+}\right)=\lim _{\tau \downarrow t} \sigma(\tau)$, for $\tau \geq 0$ in continuous time case, and $\sigma\left(t^{+}\right)=\sigma(t+1)$, for $t \geq 0$ in discrete time case, then it is called a switching signal.

A switching signal is said to be a switching path if it is defined as mapping of finite, semi-open time interval into the index set $M$ such that $\sigma:\left[t_{0}, t_{1}\right) \rightarrow M$ for every $\left[t_{0}, t_{1}\right)$ with $t_{0}<t_{1}<+\infty$. A switching law is called a time-driven switching law if it depends only on time and its past value $\sigma\left(t^{+}\right)=\sum(t, \sigma(t))$. A switching law is called a state-feedback switching law if it depends only on its past value an on the values of state variables at that time $\sigma\left(t^{+}\right)=\sum(\sigma(t), x(t))$ for $\forall t \in\left[t_{0},+\infty\right)$. A switching law is called a output-feedback switching law if it depends only on its past value an on the values of output variables at that time 
$\sigma\left(t^{+}\right)=\sum(\sigma(t), y(t))$ for $\forall t \in\left[t_{0},+\infty\right)$. At present, no other concepts and notions about feasible switching signals matter.

The concept of average dwell time is given below and the respective rather important fundamental result is cited too, both due to Hespana and Morse [9].

Definition 3 (Hespanha \& Morse, 1999): For a switching signal $\sigma$ and any $t_{2}>t_{1}>t_{0}$, let $N_{\sigma}\left(t_{1}, t_{2}\right)$ be the number of switching over the interval $\left[t_{1}, t_{2}\right)$. If the condition $N_{\sigma}\left(t_{1}, t_{2}\right) \leq N_{0}+\left(t_{2}-t_{1}\right) / \tau_{a}$ holds for $N_{0} \geq 1$, $\tau_{a}>0$, then $N_{0}$ and $\tau_{a}$ are called the average dwell time (ADT) and the chatter bound, respectively.

Theorem 1 (Hespanha \& Morse, 1999): Consider the switched system (3), and let $\alpha$ and $\mu$ be given constants. Suppose that there exist smooth functions $V_{\sigma(t)}: \mathfrak{R}^{N} \rightarrow \mathfrak{R}, \sigma(t) \in \ell$, and two $K_{\infty}$ functions $k_{1}$ and $k_{2}$ such that for each $\sigma(t)=i$, the following conditions hold:

$$
\begin{gathered}
k_{1}\left(\left|x_{t}\right|\right) \leq V_{i}\left(x_{t}\right) \leq k_{2}\left(\left|x_{t}\right|\right), \dot{V}_{i}\left(x_{t}\right) \leq-\alpha V_{i}\left(x_{t}\right), \text { and } \\
\text { for any }(i, j) \in \ell \times \ell, i \neq j, V_{i}\left(x_{t}\right) \leq \mu V_{j}\left(x_{t}\right) .
\end{gathered}
$$

Then the system is globally uniformly asymptotically stable for any switching signal with ADT $\tau_{a}>\tau_{a}^{*}=\frac{\ln \mu}{\alpha}$.

Theorem 1 in fact considers multiple Lyapunov functions with a "jump" on the switching boundary. An extension in [36], due to Ye et al (1998), the underlying essentialities of which were further highlighted in [38] by Zhang and Gao (2010), allows the Lyapunov-like function to rise to a limited extent, and in addition to the jump on switching boundary $[8,39,40]$. This is the so-called weak Lyapunov function, and it allows both the jump on the switching boundary and the increase over any time interval of interest. Now consider $\sigma(t) \in i$ and then, within the interval $\left[t_{i}, t_{i+1}\right]$, denote the unions of scattered subintervals during which the week Lyapunov function is increasing and decreasing by $T_{r}\left(t_{i}, t_{i+1}\right)$ and $T_{d}\left(t_{i}, t_{i+1}\right)$, respectively. Hence it holds $\left[t_{i}, t_{i+1}\right)=T_{r}\left(t_{i}, t_{i+1}\right) \cup T_{d}\left(t_{i}, t_{i+1}\right)$. Further let use $T_{r}\left(t_{i+1}-t_{i}\right)$ and $T_{d}\left(t_{i+1}-t_{i}\right)$ to represent the length of $T_{r}\left(t_{i}, t_{i+1}\right)$ and $T_{d}\left(t_{i}, t_{i+1}\right)$, correspondingly. Then the following important result can be obtained: 
Theorem 2 (Ye et al, 1998; Zhang \& Gao, 2010): Consider the switched system

$$
\dot{x}_{t}=f_{\sigma}\left(x_{t}\right) \text {, }
$$

and let $\alpha>0, \beta>0$ and $\mu>1$ are prescribed constants. If there exist smooth functions $V_{\sigma(t)}: \mathfrak{R}^{n} \rightarrow \mathfrak{R}$ and two $K_{\infty}$ functions $k_{1}$ and $k_{2}$ such that for each $\sigma(t)=i$, the following conditions hold:

$$
\begin{gathered}
k_{1}\left(\left|x_{t}\right|\right) \leq V_{i}\left(x_{t}\right) \leq k_{2}\left(\left|x_{t}\right|\right), \\
\dot{V}_{i}\left(x_{t}\right) \leq\left\{\begin{array}{l}
-\alpha V_{i}\left(x_{t}\right) \quad \text { over } \quad t \in T_{d}\left(t_{i}, t_{i+1}\right), \\
\beta V_{i}\left(x_{t}\right) \quad \text { over } \quad t \in T_{r}\left(t_{i}, t_{i+1}\right)
\end{array},\right. \\
V_{i}\left(x_{t}\right) \leq \mu V_{j}\left(x_{t}\right) \quad \forall\left(\sigma(t)=i \quad \& \quad \sigma\left(t^{-}\right)=j\right),
\end{gathered}
$$

Then the system is GUAS for any switching signal with ADT

$$
\tau_{a}>\tau_{a}^{s}=\frac{(\alpha+\beta) T_{\max }+\ln ^{\mu}}{\alpha}, T_{\max }=\max T_{r}\left(t_{i-1}, t_{i}\right), \quad \forall i .
$$

It may well be seen that the result above actually includes Theorem 1 as a special case. Namely, $\beta=0$ implies no increase over the interval and hence $T_{\max }=0$, then the ADT condition reduces to the ADT condition $\tau_{a}>\tau_{a}^{*}=\frac{\ln \mu}{\alpha}$, (5) in Theorem 1. It is precisely this generality of the weak Lyapunov functions that has given incentives to explore the alternatives on fast and slow switching rules [8], [31] in this study.

\section{Fast and Slow Switching Modes: Recent Discoveries}

In the sequel the derived novel results, which are subject to further exploration in this paper, are presented first. In what follows subsequently, the following notations from work [3] of Branicky (1998) are adopted throughout. In particular, a general arbitrary switching sequence is expressed by

$$
\sum=\left\{x_{0} ;\left(i_{0}, t_{0}\right),\left(i_{1}, t_{1}\right), \ldots,\left(i_{j}, t_{j}\right), \ldots, \mid i_{j} \in \bar{M}, j \in N\right\}
$$

in which $t_{0}$ is the initial time, $x_{0}$ is the initial state, $\left(i_{k}, j_{k}\right)$ means that the $i_{k}$-th subsystem is activated for $t \in\left[t_{k}, t_{k+1}\right)$. Therefore, when $t \in\left[t_{k}, t_{k+1}\right)$, the 
trajectory of the switched system (1) is produced by the $i_{k}$-th subsystem. Thus, for any $j \in \bar{M}$, the set

$$
\sum_{t}(j)=\left\{\left[t_{j_{1}}, t_{j_{1}+1}\right), \cdots\left[t_{j_{n}}, t_{j_{n}+1}\right) \cdots \sigma(t)=j, t_{j_{k}} \leq t \leq t_{j_{k}+1}, k \in N\right\}
$$

denotes the sequence of switching times of the $j$-th subsystem, in which the $j$-th subsystem is switched on at $t_{j_{k}}$ and switched off at $t_{j_{k}+1}$.

\section{A Novel Insights into the Complexity of Switching}

It should be noted, Theorem 2 is a slow switching result in the sense that it is characterized by a lower bound on the average dwell time. Recently, the following two novel results have emanated from the novel insights into the switching complexity.

Theorem 3 [8, 31] Consider the switched system

$$
\dot{x}_{t}=f_{\sigma}\left(x_{t}\right) \text {, }
$$

and let $\alpha>0, \beta>0$ and $\mu>1$ are prescribed constants. If there exist smooth functions $V_{\sigma(t)}: \Re^{n} \rightarrow \mathfrak{R}$ and two $K_{\infty}$ functions $k_{1}$ and $k_{2}$ such that for each $\sigma(t)=i$, the following conditions hold:

$$
\begin{gathered}
k_{1}\left(\left|x_{t}\right|\right) \leq V_{i}\left(x_{t}\right) \leq k_{2}\left(\left|x_{t}\right|\right), \\
V_{i}\left(x_{t}\right) \leq \mu V_{j}\left(x_{t}\right) \quad \forall\left(\sigma(t)=i \quad \& \quad \sigma\left(t^{-}\right)=j\right), \\
\dot{V}_{i}\left(x_{t}\right) \leq\left\{\begin{array}{l}
-\alpha V_{i}\left(x_{t}\right) \quad \text { over } \quad t \in T_{d}\left(t_{i}, t_{i+1}\right) \\
\beta V_{i}\left(x_{t}\right) \quad \text { over } \quad t \in T_{r}\left(t_{i}, t_{i+1}\right) .
\end{array}\right.
\end{gathered}
$$

Then the system is GUAS for any switching signal with ADT

$$
\tau_{a}<\tau_{a}^{f}=\frac{(\alpha+\beta) T_{\min }-\ln ^{\mu}}{\beta}, \quad T_{\min }=\min T_{d}\left(t_{i-1}, t_{i}\right), \forall i
$$

Intuitively, a switched nonlinear will achieve induced stability by arbitrary switching if the upper bound for the fast switching is larger than the lower bound for slow switching [20. This is the essence of the subsequent theorems.

Theorem $4[8,31]$ Consider the switched system 


$$
\dot{x}_{t}=f_{\sigma}\left(x_{t}\right),
$$

and let $\alpha>0, \beta>0$ are prescribed constants. If there exist smooth functions $V_{\sigma(t)}: \mathfrak{R}^{n} \rightarrow \mathfrak{R}$ and two $K_{\infty}$ functions $k_{1}$ and $k_{2}$ such that for each $\sigma(t)=i$, the following conditions hold:

$$
\begin{gathered}
k_{1}\left(\left|x_{t}\right|\right) \leq V_{i}\left(x_{t}\right) \leq k_{2}\left(\left|x_{t}\right|\right), \\
V_{i}\left(x_{t}\right)=V_{j}\left(x_{t}\right) \quad \forall\left(\sigma(t)=i \quad \& \quad \sigma\left(t^{-}\right)=j\right), \\
\dot{V}_{i}\left(x_{t}\right) \leq\left\{\begin{array}{l}
-\alpha V_{i}\left(x_{t}\right) \quad \text { over } \quad t \in T_{d}\left(t_{i}, t_{i+1}\right), \\
\beta V_{i}\left(x_{t}\right) \quad \text { over } t \in T_{r}\left(t_{i}, t_{i+1}\right) .
\end{array}\right.
\end{gathered}
$$

Then the system is GUAS for arbitrary switching signal if the following conditions are fulfilled:

$$
\frac{T_{\max }}{T_{\min }} \leq \frac{\alpha}{\beta}, T_{\max } \equiv \max T_{r}\left(t_{i-1}, t_{i}\right), T_{\min } \equiv \min T_{d}\left(t_{i-1}, t_{i}\right), \quad \forall i .
$$

Theorem $5[8,31]$ Consider the switched system

$$
\dot{x}_{t}=f_{\sigma}\left(x_{t}\right)
$$

and let $\alpha>0, \mu>1$ be given constants. Suppose that there exist $C^{1}$ functions $V_{\sigma(t)}: \Re^{N} \rightarrow \Re, \sigma(t) \in \ell$, and two $K_{\infty}$ functions $k_{1}$ and $k_{2}$ such that $\forall \sigma(t)=i, \quad k_{1}\left(\left|x_{t}\right|\right) \leq V_{i}\left(x_{t}\right) \leq k_{2}\left(\left|x_{t}\right|\right), \quad \dot{V}_{i}\left(x_{t}\right) \leq-\alpha V_{i}\left(x_{t}\right), \quad$ and $\forall(i, j) \in \ell \times \ell, i \neq j, V_{i}\left(x_{t}\right) \leq \mu V_{j}\left(x_{t}\right)$.

Then the system is GUAS for any switching signal if and only if the ADT satisfies the condition $\tau_{a}>\tau_{a}^{*}=\frac{\ln \mu}{\alpha}$.

The details of poofs for the above presented results are found in [8]. It is important to notice that the slow switching condition reduces to the average dwell time of Hespanha and Morse precisely, as they have defined it in their 1999 seminal paper [12].

\section{B Novel Supporting Evidence via Simulation Experiments}

At this point, let recall the expression of a general arbitrary switching sequence, namely: 


$$
\sum=\left\{x_{0} ;\left(i_{0}, t_{0}\right),\left(i_{1}, t_{1}\right), \ldots,\left(i_{j}, t_{j}\right), \ldots, \mid i_{j} \in \bar{M}, j \in N\right\}
$$

Also, notice that $t_{0}$ is the initial time, $x_{0}$ is the initial state, $\left(i_{k}, j_{k}\right)$ means that the $i_{k}$-th subsystem is activated for $t \in\left[t_{k}, t_{k+1}\right)$. Duplicate the template file by using the Save As command, and use the naming convention prescribed by your conference for the name of your paper.

Next, let recall that by means of Jacobians the basic linearization of general nonlinear systems, such as the class of time-varying nonlinear plant processes to be controlled, is as follows:

$$
\overrightarrow{\dot{x}}(t)=\vec{f}(\vec{x}(t), \vec{u}(t) ; t), \vec{x}\left(t_{0}\right)=\vec{x}_{0}, \vec{y}(t)=\vec{g}(\vec{x}(t) ; t) .
$$

Upon linearization of nonlinear functions $\vec{f}, \vec{g}$ in the vicinity neighborhood of a certain operating state, e.g. such as a steady-state operating point $E P\left(\vec{u}_{c}, \vec{x}_{c}\right.$, $\vec{y}_{c}$ ) with the steady-states $\vec{x}_{c}, \vec{y}_{c}$ which may happen under some steady control input $\vec{u}_{c}$, model (6) yields

$$
\left\{\begin{array}{c}
\overrightarrow{\dot{x}}(t)=A(t)_{n \times n} \vec{x}(t)+B(t)_{n \times r} \vec{u}(t), \quad \vec{x}\left(t_{0}\right)=\vec{x}_{0}, \\
\vec{y}(t)=C(t)_{m \times n} \vec{x}(t),
\end{array}\right.
$$

where matrices $\quad A(t)_{n \times n}=\partial \vec{f} / \partial \vec{x}_{\left.\right|_{x_{c}, u_{c}}}, \quad B(t)_{n \times r}=\partial \vec{f} / \partial \vec{u}_{x_{c}, u_{c}}$, $C(t)_{n \times r}=\partial \vec{g} / \partial \vec{x}_{\left.\right|_{c}, u_{c}}$. Should the steady-state operating point $E P\left(\vec{u}_{c}, \vec{x}_{c}, \vec{y}_{c}\right)$ is a desired equilibrium (which usually is in practice) that can be achieved under a certain synthesized equilibrium control vector $\vec{u}_{c}=\vec{u}_{c}^{e}=$ const, defining the desired equilibrium state vector $\vec{x}_{c}=\vec{x}_{c}^{e}=$ const and hence the desired steadystate equilibrium output vector $\vec{y}_{c}=\vec{y}_{c}^{e}=$ const , then model (6) yield

$$
\overrightarrow{\dot{x}}=A_{n \times n} \vec{x}(t)+B_{n \times r} \vec{u}(t), \vec{x}\left(t_{0}\right)=x_{0} ; y(t)=C_{n \times m} \vec{x}(t) .
$$

It is therefore that the Lyapunov asymptotic stability requirement on the system's steady-state equilibrium is indispensable and rigorous requirement. It is this requirement precisely which is being enhanced by involving a switching law in addition to the synthesized feedback control. The presented illustrations obtained by means of simulated time-responses further below, along with the above theoretical results, highlight the concepts essence of both the fast and slow switching as well as the average dwell time. Also, they emphasize the importance of achieving as- 
ymptotic Lyapunov stability under arbitrary switching as well as under averagedwell time switching. In any case, these findings guarantee in the close loop the ultimately uniform bounded operation of the plant at desired equilibrium steadystate shall be reached despite the possible uncertainties of plant model.

For this purpose let now consider the application of the arbitrary switching sequence (5) according to average-dwell time principle to a second-order twoinput-two-output, uncertain nonlinear plant system of class (6), whose states are detectable and measurable. (If they are not detectable and measurable., then state estimator ought to be employed in composite control strategy combining estimated state feedback and average-dwell time principle). Further, let assume the respective Jacobian state-transition and output subsystems represent a controllable and observable linearized system model in the vicinity of the desired steady-state operating equilibrium.

Thus, let consider the following example [8] for which an uncertain plant of class (6) yields system (7 b) having the system matrices:

$$
\begin{gathered}
A_{11}=\left[\begin{array}{cc}
-5 & 4 \\
0 & 2
\end{array}\right], A_{2}=\left[\begin{array}{cc}
-15 & 1 \\
0 & -10
\end{array}\right], A_{21}=\left[\begin{array}{cc}
2 & 0 \\
1 & -5
\end{array}\right], A_{22}=\left[\begin{array}{cc}
-15 & 0 \\
-5 & -4
\end{array}\right], \\
B_{11}=B_{12}=\left[\begin{array}{ll}
1 & 1 \\
1 & 1
\end{array}\right], B_{21}=B_{22}=\left[\begin{array}{ll}
3 & 1 \\
1 & 5
\end{array}\right],(8 \mathrm{~b}) \\
C_{11}=C_{12}=\left[\begin{array}{ll}
1 & 1 \\
1 & 1
\end{array}\right], C_{11}=C_{12}=\left[\begin{array}{ll}
1 & 1 \\
1 & 1
\end{array}\right] .(8 \mathrm{c})
\end{gathered}
$$

Next, let define region subsets

$$
\Omega_{1} \cup \Omega_{2} \equiv\left\{\begin{array}{l}
\Omega_{1}=\left\{x(t) \in R^{n} \mid x(t)^{\mathrm{T}}\left(P_{1}-P_{2}\right) x(t) \geq 0, x(t) \neq 0\right\}, \\
\Omega_{2}=\left\{x(t) \in R^{n} \mid x(t)^{\mathrm{T}}\left(P_{2}-P_{1}\right) x(t) \geq 0, x(t) \neq 0\right\} .
\end{array}\right\}
$$

where matrices $P_{1}, P_{2}$ are designed in the course of stabilizing state feedback control synthesis using LMI as in most of previous works on switched systems and control, which were surveyed here in the introduction. In particular, see work [43]. It is therefore that system's state space is generated by $\Omega_{1} \cup \Omega_{2}=R^{n} \backslash\{0\}$. The closed-loop system therefore should be asymptotically stable or uniformly bounded at least due to the switching law

$$
\sigma(t)=\left\{\begin{array}{cc}
1 & x(t) \in \Omega_{1}, \\
2 & x(t) \in \Omega_{2} \backslash \Omega_{1} .
\end{array}\right\}
$$


regardless of the plant uncertainty. The obtained computer simulation results are depicted in Figure 3 and Figure 4. In the former figure there are presented the switching sequences employed, while in the latter figure there are presented the state responses of the controlled plant process.



(a)

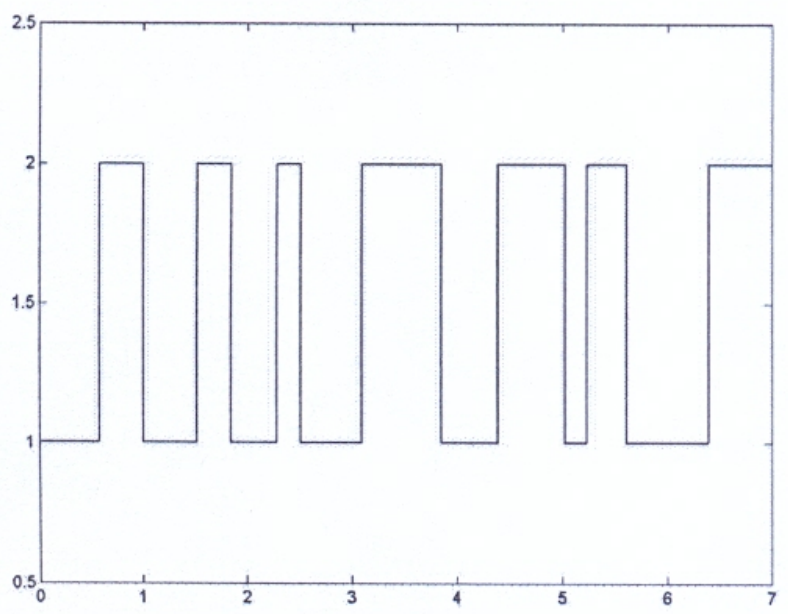

(b)

Figure 3 Evolution of the time responses for the considered class of switching sequences demonstrating the fast (a) and the slow (b) switching modes with the average-dwell-time strategy, in conjunction with the state-feedback controlled transitions in Fig. 3, which converge to the equilibrium state in step-wise mode with continuously convergent trend.

In fact, Figure 3 depicts two typical switching sequences combining consistently fast and slow switching of two different modes, which thus demonstrate asymptotic stability can be achieved under arbitrary switching law over two consistent state zones. 
The corresponding simulation results for the plant's state vector response, when the initial conditions of states are $x(0)=[-3,1]^{T}$, have been found as depicted in Figure 4.

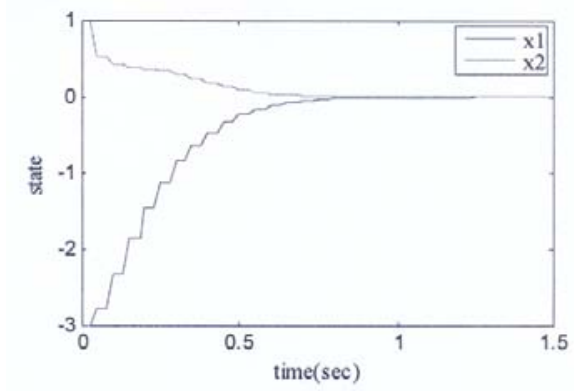

Figure 4 Fast step-wise stable evolution of the time responses of both states towards the plant's equilibrium state in closed loop under switching based state feedback control with switching laws in Fig. 3 b, which combine consistently fast and slow switching modes stability.

Before summarizing this study, let observe another similar system with uncertainties handled by means of the same procedure employing region subsets (9) and the switching law (10). Again, $P_{1}, P_{2}$ were designed in the course of stabilizing state feedback control synthesis using LMI and a smoothing filter was associated with it. The system's state space is thus generated by $\Omega_{1} \cup \Omega_{2}=R^{n} \backslash\{0\}$. The simulation results for the plant's state vector response in this case, when the initial states were chosen as $x(0)=[+2,-2]^{T}$, have been found as depicted in Figure 5.

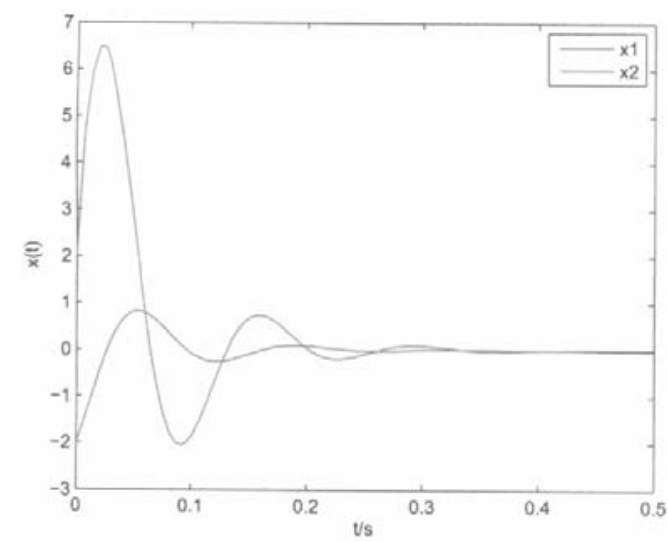

Figure 5 Fast step-wise stable evolution of the time responses of both states towards the plant's equilibrium state in closed loop under switching based state feedback control with switching laws in Fig. $3 \mathrm{~b}$, which combine consistently fast and slow switching modes stability along with the filter's smoothing effect. 
The computer simulation results have been obtained via Matlab-Simulink computing tools of MathWorks Co [23, 24] on the grounds of using Linear Matrix Inequalities [2], [10]. At this point again it should be noted, as R. W. Brocket has emphasized in article [4], the methodology of Linear Matrix Inequalities (LMI) is based on the seminal mathematical and systems-theoretical discoveries by V. A. Yakubovich for nonlinear control systems. These include not only matrix inequalities [28-29] but also the rather essential S-procedure for analysis and synthesis of nonlinear control systems [30]. These seminal results by Yakubovich have enabled the emergence of the respective works [2], [10] by S. P. Boyd and co-authors (1994) and by P. Gahinet (1995).

\section{Concluding Remarks and Future Research}

This paper has presented further investigation and supportive evidence to the novel characterization of nonlinear switched systems via adopting constrained switching law over two consistent state zones through concepts of slow and fast switching. These concepts were initially defined in works [8], [31]. It should be noted, a fast switching rule may even guarantee globally uniform asymptotic stability of the desired steady-state equilibrium, to which some synthesized statefeedback control has driven the controlled plant process. These findings thus prove that the standard average dwell time condition associated with the multiple Lyapunov functions, in fact, appears to be a kind of if and only if condition. It should be noted, the above presented results seems to have extended further the existing insight, knowledge and understanding of stability theory for switched nonlinear systems and control. In addition, also it has been highlighted why the issue of system stability for switched nonlinear systems is more complicated in comparison with the non-switched systems even when the switching involves average-dwell time. It is therefore that inevitably control systems for cases of complex interconnected plants even when average-dwell time switching principle is employed along with more traditional state feedback become control systems beyond decentralized control.

Apparently though, in such complex control systems, the system dynamics phenomenology become even more complex if observer-based output feedback strategy ought to be employed along with average-dwell time switching law feedbacks. Thus this brief outline tackling of these issues seems to have defined a new direction for future research in which there several paths to follow. However, proper definition of tasks to be explored in the near future is yet to be elaborated on and precisely defined in mathematical terms since it involves not only uncertainties but also information constraints. For this purpose, notice the recent work [29] by Sun and co-authors (2019), where an idea on how to approach the stateconstrained case was developed within context of switched nonlinear systems. Another idea is found in works [17, 18] by Li and co-authors (2017 a, b) in which impulsive observers for switched nonlinear systems were instrumental. On the 
other hand, it is also instrumental to notice recent work [1] by Andriyevsky and co-authors (2010) who suggested a way towards unified theory of control, computation and communications.

\section{Acknowledgements}

This research has been generously supported by China Postdoctoral Science Foundation (grant No. 20100471336), Beijing, and NUAA Research Funding Projects (grants No. S0985-021 and No. 1002-56YAH11011), Nanjing, P. R. of China. In part, it was also supported by a special Fund for China-Macedonia cooperation of Ministry of Education \& Science of R. of Macedonia (grant No. 143154/1), a special Fund for Turkey-Russia co-operation of Turkish Agency TUBITAK and Russian Foundation for Basic Research (grant TUBITAK-RFBR 05.09-06.09.2012/1) as well as by Fund for Science of Dogus University. Here Georgi Dimirovski and Jiqiang Wang want to acknowledge their former doctoral students, respectively, for carrying out computer simulations. They would like also to mention some inspiring question that emerged while discussing on the exciting area of switched systems and switching based control for nonlinear dynamic plants.

\section{References}

1. Andriyevsky, B. R., Matveev, A. S., and Fradkov, A. L.: Control and estimation under information constraints: Toward a unified theory of control, computation and communications (in Russian). Avtomatika i Telemechanika, 71 (4), 34-99 (2010).

2. Boyd, S. P., El Ghaoui, L., Feron, E., and Balakrishnan, V.: Linear Matrix Inequalities in Systems and Control Theory, SIAM Studies in Applied Mathematics, vol.15. The SIAM, Philadelphia, PA, USA (1994).

3. Branicky, M. S.: Multiple Lyapunov functions and other analysis tools for switched and hybrid systems. IEEE Transactions on Automatic Control, 43 (4), 475-482 (1998).

4. Brockett, R. W.: The wider influence of the work of V. A. Yakubovoch, International Journal of Robust and Nonlinear Systems, 17 (5-6), 363-368 (2007).

5. Dayawansa, W. P., and Martin, C. F.: A converse Laypunov theorem for a class of dynamical systems which undergo switching. IEEE Transactions on Automatic Control, 44 (4), 751-760 (1999).

6. DeCarlo, R. A., Branicky, M. S., Petterson, S., and Lenartsson, B.: Perspectives and results on the stability and stabilizability of hybrid systems. Proceedings of the IEEE, 88 (7), 1069-1082 (2000).

7. Dimirovski, G. M., Gough, N. E., and Barnett, S.: Categories in systems and control theory. International Journal of Systems Science, 8 (9), 1081-1090 (1977).

8. Dimirovski, G. M., Wang, J., and Yue, H.: Complexity of constrained switching for switched nonlinear systems with average dwell time: Novel characterization. In Proceedings SMC2016 of the IEEE International Conference on Systems, Man, and Cybernetics (General Chair Imre J. Rudas, Technical Program Chair 
Shun-Feng Su), Budapest, HU, 9-12 October. The IEEE, Piscataway, NJ, USA, pp. 2376-2381 (2017).

9. Emelyanov, S. V.: Variable Structure Control Systems (in Russian). Nauka, Moscow, USSR (1967).

10. Gahinet, P., Nemirovskii, A., Laub, A. J., and Chilali, M.: The LMI Tool Box. Natick: NJ: The MathWorks, Inc. (1995).

11. Hespanha, J. P.: Uniform stability of switched linear systems: Extensions of Lassalle's invariance principle. IEEE Transactions on Automatic Control, 49 (4), 470-482 (2004).

12. Hespanha, J. P. and Morse, A. S.: Stability of switched systems with average dwell time. In Proceedings of $38^{\text {th }}$ Conference on Decision and Control (General Chair E. W. Kamen; Program Chair Ch. Casandras), Phoenix, AZ, 7-10 December. The IEEE, Piscataway, NJ, USA, pp. 2655-2660 (1999).

13. Khalil, H. K.: Nonlinear Systems ( $3^{\text {rd }}$ Edition). Prentice Hall, Upper Saddle River, NJ, USA (2002).

14. Kuntsevich, V. M., and Lychak, M. M.: Synthesis of Systems of Automatic Control with the Aid of Lyapunov Functions (in Russian). Nauka, Moscow, USSR (1977).

15. Kuntsevich, V. M.: Control Under Uncertainty: Guaranteed Results in Control and Identification (in Russian). Naukova Dumka, Kyiev, UA (2006).

16. Kuntsevich, V. M., Gubarev, V. F., Kondratenko, Y. P., Lebedev, D. V., Lysenko, V. P., Editors: Control Systems Theory and Applications, Rover Publishers, Gistrup, DK - Delft, NL (2018).

17. Li, J., Ma, R., Dimirovski, G. M., and Fu, J.: Dwell-time based stabilization of switched linear singular systems with all unstable modes. Journal of the Franklin Institute: Engineering and Applied Mathematics, 354 (7), 2712-2724 (2017 a).

18. Li, J., Ma. R., and Dimirovski, G. M.: Adaptive impulsive observers for a class of switched nonlinear systems with unknown parameter. Asian Journal of Control, 19 (3), 1153-1163 (2017 b)

19. Li, L.-L., Zhao, J., Dimirovski, G. M.: Observer-based reliable exponential stabilization and $\mathrm{H}_{\mathrm{inf}}$ approach for switched systems with faulty actuators: An average dwell-time approach. Nonlinear Analysis: Hybrid Systems, 5, 479-491 (2011).

20. Li, T.-F., Dimirovski, G. M., Liu, Y., and Zhao, J.: Improved stability of a class of switched neutral systems via Lyapunov-Krasovskii functionals and an average dwell time scheme. International Journal of Systems Science, 44(6), 1076-1088 (2013)

21. Liberson, D., and Morse, A. S.: Basic problems in stability and design of switched systems. Control Systems Magazine, 19 (1), 59-70 (1999).

22. Liberzon, D.: Switching in Systems and Control. Birkhauser, Boston, MA (2003).

23. MathWorks, MATLAB. Natick, NJ: The MathWorks, Inc. (1994).

24. MathWorks, SIMULINK. Natick, MA: The MathWorks, Inc. (1999).

25. Molchanov, A. P., and Pyatnitskiy, Y. S.: Criteria for asymptotic stability of differential and difference inclusions encountered in control theory. Systems and Control Letters, 13 (1), 59-64 (1989). 
26. Morse, A. S., Supervisory control of families of linear set-point controllers: Pt.1 Exact matching; and Pt. 2 Robustness. IEEE Transactions on Automatic Control, 41(10), 1413-1431 (1996); 42(10), 1500-1515 (1997).

27. Sworder, D. D.: Control of systems subject to sudden changes. Proceedings of the IEEE, 64 (8), 1219-1225 (1976).

28. Sun, X. M., Zhao, J., and Dimirovski, G. M.: State feedback control for discrete delay systems with controller failures based on average-dwell time method. IET Control Theory and Applications, 2 (2), 126-132 (2008).

29. Sun, Y., Zhao, J., and Dimirovski, G. M.: Adaptive control for a class of stateconstrained high-order switched nonlinear systems with unstable subsystems. Nonlinear Analysis: Hybrid Systems, 32, 91-105 (2019).

30. Sun, Z., and Ge, S. S.: Analysis and synthesis of switched linear control systems. Automatica, 41 (2), 181-185 (2005).

31. Wang, J., Hu, Z., Yue, H., Dimirovski, G. M.: Two characterizations of switched nonlinear systems with average dwell time. International Journal of Applied Mathematics and Machine Learning, 4 (2), 81-91 (2016).

32. Xiang, J., and Xiao, W.: Stabilization of switched continuous-time systems with all modes unstable via dwell-time switching. Automatica, 50 (3), 940-945 (2014).

33. Yakubovich, V. A.: Solution to some matrix inequalities encountered in the theory of automatic control (in Russian). Dokladi Akademii Nauk SSSR, 143 (6), 1304-1307 (1962).

34. Yakubovich, V. A.: Solution to some matrix inequalities encountered in the nonlinear theory of automatic control (in Russian). Dokladi Akademii Nauk SSSR, 156 (2), 278-281 (1964).

35. Yakubovich, V. A.: S-procedure in the nonlinear theory of automatic control (in Russian). Vestnik Leningradskogo Gosudarstvenogo Universsiteta, Seriya Matematika, Mechanika, Astronomiya, $162-77$ (1971).

36. Ye, H., Michel, A. N., and Hou, L.: Stability theory for hybrid dynamical systems. IEEE Transactions on Automatic Control, 43 (4), 461-474 (1998).

37. Zhai, G. S., Hu, B., Yasuda, K., and Michel, A. N.: Piecewise Laypunov functions for switched systems with average dwell time. Asian Journal of Control, 2 (3), 192-197 (2000).

38. L., Zhang, L., and Gao, H.: "Asynchronously switched control of switched linear systems with average dwell time.” Automatica, 46 (9), 953-958 (2010).

39. Zhao, J., and Nie, H.: Sufficient conditions for input-to-state stability of switched systems. Acta Automatica Sinica, 29 (2), 252-257 (2003).

40. Zhao, J., and Dimirovski, G. M.: Quadratic stability of switched nonlinear systems. IEEE Transactions on Automatic Control, 49 (4), 574-578 (2004).

41. Zhao, J. and Hill, D. J.: Passivity and stability of switched nonlinear systems. Systems and Control Letters, 57 (2), 158-164 (2008).

42. Zhao, J. and Hill, D. J.: Dissipativity theory for switched systems. IEEE Transactions on Automatic Control, 53 (4), 941-953 (2008).

43. Zhao, S., Dimirovski, G. M., and Ma, R.: Robust $\mathrm{H}_{\text {inf }}$ control for non-minimum phase switched cascade systems with time delay. Asian Journal of Control, 17 (4), 1-10 (2015). 